\title{
ICT and Special Educational Needs: Using Meta-synthesis for Bridging the Multifaceted Divide
}

\author{
Ying Liu ${ }^{1}$, Annita Cornish ${ }^{1}$, and June Clegg ${ }^{2}$ \\ ${ }^{1}$ Homerton College, Cambridge University, Hills Road, Cambridge CB2 2PH UK \\ \{y1317, ac550\}@cam.ac.uk \\ ${ }^{2}$ New Hall College, Cambridge University, Huntingdon Road, Cambridge CB3 ODF UK
}

\begin{abstract}
We elicit some critical views on how the applications of information communication technology (ICT) can be approached for people with special educational needs (SEN). The findings, based on a number of concrete casestudies, clearly confirm that, despite of the huge research and developmental effort having been made for advancing ICT applications in education, ICT as a whole is surprisingly lacking a systematic discourse with educational domains, even much less concerning those who have learning difficulties. Whilst well known technical tools such as word-processors, screen-reading software and problem-solving software packages still provide useful "snapshots" of ICT applications from particular points in time, we argue that these pictures now require updating. To enable the ICT development crossing multi-disciplinary boundaries, a meta-synthesis approach is innovated. The approach consists of four inter-operated components through ICT, i.e., assistive, sensory, communicative and interactive component.
\end{abstract}

Keywords: Meta-synthesis, Digital divide, e-Inclusion, Complex problemsolving, Education, Special educational needs.

\section{Introduction}

The term "special educational needs" (SEN) covers many kinds of significant learning difficulties that learners can have [1], relating to a range of problems from particular impairments [2], to the complex interaction between the learners and related school, ethnic, social, political, cultural factors [3][4].

Special education is therefore a field involved in a theoretical as well as empirical enquiry on all the factors that cause significant learning barriers experienced by some learners compared with other similar learners, and seeking active intervention and practical procedures that can be used to enable people with SEN better learning in a typical setting such as a school, home, social and/or virtual community [5].

To date, the results of national and international investments in ICT for education have been the major driving forces of the e-learning transformation; so have been the regional, national and international initiatives established for integrating ICT within special education. E.g., the British Educational and Communications Technology Agency (Becta) leads agencies in supporting the government's programme to develop National Grid for Learning (NGfL). It has a new and clear remit to ensure NGfL 
developments take account for SEN and the teachers [6]. The Irish National Centre for Technology in Education published a report shows that the interest among teachers in using ICT with students with SEN is very high. The centre sets up sites for Suppliers, Section of SEN, SEN Technology, Software Central and Curriculum Resources [7].

There are varieties of commercial products for SEN as well. As easily found on the Web, the software developers claim to offer professional resources, special needs software and assistive technology, such as a) software and typing books for students with cerebral palsy, missing fingers, learning disabilities, dyslexia, visually impaired, etc.; b) learning solutions for classrooms and homes for kids, and c) accessibility software and text to speech software. However, how to evaluate such a toolkit is outside of the scope of this paper. Nevertheless this is an important research area. See, e.g. Woodward et al examined the research on software curriculum designed specifically for pupils with SEN [8].

Here the primary concern is that, ICT itself can create significant barriers for learners and teachers. Williams et al [9] conclude that the current ICT for education surprisingly lacks research into the usability of the various applications developed, even less concerning those with learning difficulties. Mooij et al conclude that the use of ICT in general merely showed characteristic of traditional approaches to learning, after interviewed 331 teachers in the highest grade of primary education. The methods employed by teachers to adapt education to the needs and abilities of individual pupils proved quite limited [10].

With the mixed views given above and more others to be discussed further, we argue, in section 2, that it is equivalently crucial to innovate a meta-synthesis approach that enables deeper understandings of multi-disciplinary uses of ICT for SEN. In section 3 we introduce the areas of need, i.e. "communication and interaction", "sensory and/or physical", "cognition and learning", and "behaviour, emotional and social development". We synthesis the uses of ICT in the first and second area in section 4 and give conclusions in section 5 .

\section{Bridging ICT and SEN - Converging or Accommodating the Needs?}

As noted, ICT and SEN are complex research areas; Linking the two, however, can be even more difficult. A great deal of knowledge within the area of special education is mainly based on how the practitioners understand their own work. Bridging ICT and SEN should receive more attentions beyond the studies on a basis of either accepting or rejecting ICT. To do this, we use meta-synthesis as our analytical tool.

Meta-synthesis methodology has been widely employed in solving many kinds of problems: from the concern for classic engineering analysis, such as the flow of matter and/or energy, to the concern for modern-day controlling communications, such as information. Hence, for a research strategy, it forces one to look at a problem in its entirety. Meta-synthesis method is a powerful way of thinking to tackle problems of complex, open and giant systems. The method is formulated by Chinese scientists Qian, X.S. and his colleagues [12][13]; it emphasizes the synthesis of acquisitioning knowledge from human experts and integrating quantitative methods with qualitative knowledge. Later it is evolved into meta-synthetic engineering which emphasises the 
discourse among research fields in studying human, society, information and communication technologies. At present, we do not seem having such a systematic discourse between ICT and SEN. Yet complex problems are emerging in SEN: a) new learning environments with different teaching styles, b) equity in access to ICT and c) an ongoing infusion of new ICT oriented opportunities.

ICT covers a large variety of technologies in any form of a product, system, network, tool, solution, method or technique that stores, retrieves, processes, manipulates, displays, and/or transmits data electronically, e.g. a PDA (personal digital assistant), handheld computer, wireless internet, mobile WWW, smart gadget, broadband, sensory device or robot. Evidently, the new digital technologies have transformed the way we live and work. This has become the reality of the information revolution. Many believe that ICT is now transforming the way we learn, along with our needs for leaning [14]. There have been earlier communication revolutions - print being a primary example. It took centuries for the printing press to impact on living, working and learning. The current digital changes are taking place only within a generation even within a decade. When change takes place so quickly, it happens very unevenly.

Since here the major emphasis given on ICT is assisting the learners with SEN, it must be noted however, that not all impairments or disabilities create barriers to learning. However, there are conditions and impairments that are known to create the significant barriers, unless accommodations are made. Enabling special education through the uses of ICT becomes increasingly important. Such importance is clearly indicated by several existing journals in subject of "special educational technology" or even "educational technology" in general.

Up to date, an accommodation of persons with SEN is based on a geo-located institution such as a school. The accommodation relies on instruments of school systems, laws, policies, legislations, initiatives, frameworks, guidelines, trainings and teaching practice to adapt to the learners. The best instance for illustrating this approach for accommodating SEN is perhaps the UK government policy that has tended to favour models of inclusive education that promote a process of increasing participation and decreasing the exclusion of vulnerable students from the culture, curricula and communities of local schools. The Audit Commission examined how the 1981 and 1996 Special Educations were working [15]. It reported that schools were struggling. A considerable amount of difficulties still exist in enabling inclusive policies today. To this extent, inclusive policy presents an even more confused message and causes the closure of special schools and "forcing" some children into mainstream schools when it is not in their best interests to be there, resulting in distress for pupils and parents. Thus, alternative approaches, strategies or methods must be sought beyond such an accommodation. Providing opportunities to receive an education without being accountable for ensuring educational outcomes simply perpetuates inequity in a more subtle form.

Hence we argue, from a meta-synthesis point of view, that it is a convergence that brings multi-disciplinary resources together physically or virtually; It is such a convergence that provides opportunities where solutions can be found for SEN wherever the learners and teachers are placed. For instance, children with speech and language communication needs benefit from mainstream education with additional support mechanisms, especially in the early years, but not extending into secondary educations; There is evidence that the multi-method approach is promising. Researchers 
report that a combination of strategies produces more powerful effects than a single strategy solution. For children with communication and interaction difficulties associated with severe and profound learning difficulties, intervention aims may vary from bringing the child's language skills up to an age equivalent level, social interaction with peers, using basic cognitive processes to develop information handling and management within the curriculum, removing obstacles to enable the child to participate in learning [16].

Moreover, our meta-synthesis approach views that the question is no longer how to meet individual needs at one institution, but in which environment is this person likely to learn and by what means is this person likely to get supports. In principle, ICT has the capacity to create virtual spaces and dissolve boundaries, whether between countries or between a subject, teacher and learner. But ICT itself may not make an accommodation for users. Others call this area of research as inclusive design, universal design, user adaptation, adaptive user interface, or e-inclusion. Simple examples are changing the size of the text, the colour, or adding speaking browser or special keyboards.

\section{The Learning Contexts}

Many researchers have also usefully reviewed ICT in SEN and provided the knowledge bridging ICT and SEN to some extent, demonstrating many useful insights of ICT applications for the teachers and the kind of leaning they want to foster [17]. However we found that it is necessary to take a more systematic approach to investigate the uses of ICT for the following three reasons.

First, we found that many ICT innovations have occurred where technology may assist people with SEN, but may not yet be explored. Product engineering is evolving from stand-alone devices and applications to distributed, connected, integrated, and multi-technology systems. Electronic products are becoming smart and software systems are becoming adaptive and personalized. The movement toward smaller, easier to use, micro-technologies, with larger-scale integration, increased performance, and reduced price not only benefits the general population, but also may have the potential to benefit those with SEN. Many exemplary ICT practices and case studies have been reported, but these developments may require to be usefully generalised.

Second, the subject of ICT in special education also refers to one of the most influential changes in our educational systems - a change process that is not only going to determine a classroom culture, a new teaching experience, a new training programme, a new policy, or a technological innovation, but also the nature of learning and the teaching process and hence the nature of coming generations of special educational systems.

Third, systematic views on current SEN in respect to learning and teaching are established. The "areas of need" as defined in the 2001 SEN Code of Practice are used as essential categories here to enable this investigation [18]; they are: Communication and Interaction, Sensory and/or Physical, Cognition and Learning, Behaviour, Emotional and Social Development. Within the each area, teaching strategies, types of the learners, as well as the ways and barriers where the teachers and learners communicate are identified. The sections below are within these contexts. 


\section{Converging the Needs Through ICT}

So what is now needed is to follow to identify the greater ICT challenges.

\subsection{For Assisting the Learners}

Many utilize assistive technologies to enhance functioning in activities of daily living, control of the environment, positioning and seating, vision, hearing, recreation, mobility, reading, learning and studying math, motor aspects of writing, composition of written material, communication, and computer access. Technologies used range from low-tech devices, such as pictorial communication boards or adapted eating utensils, to high-tech devices including adapted software and voice output devices with speech synthesis.

The uses of ICT in SEN can be approached by assistive technology (AT). AT is commonly accepted as the technology designed to enable and improve access a range of services for disabled users. In contexts of SEN, AT is referred to a set of aids in a form of device, hardware, software and network system, to enable the learners to access classes, practical sessions, informal/optional study skills sessions, distance learning, libraries, learning centres, etc.

\subsection{For Sensory and Physical Access}

The Joint Information Systems Committee (JISC) of the UK's Higher and Further Education Funding Councils has been involved for some years in supporting those responsible for ensuring appropriate access to electronic information services for those with disabilities. Since 2001, JISC has funded the TechDis service. A list of the hardware, software and network systems that can be used within SEN contexts can be found with links in TechDis Accessibility Database [19].

To use hardware for instances, CCTV and magnifying systems can assist students to magnify library books and other documents. There are alternative in-put devices such as trackballs, touch-pads and specialist keyboards for mobility impaired students and those with dexterity difficulties who may need adaptations to a conventional mouse or keyboard. Workstations are adjustable or high enough for wheelchair users and a good selection of ergonomic items to support those with back problems, RSI and encourage safe computer use.

To use software for instances, a screen reader software enables blind and visually impaired students to work with computers, listen to spoken descriptions of text-based online content such as web sites, intranets and virtual learning environments, as long as the content has been designed to meet W3C WAI guidelines. Spell checkers, word prediction, text to speech and speech recognition can be particularly suitable for students with dyslexia and other learning difficulties. Speech recognition software can also assist those with those with mobility and dexterity difficulties. Accessible features of Windows and Office products have made many accessible options built in to Microsoft products. These can be accessed by clicking on the START button and then going to Programs $>$ Accessories $>$ Accessibility. These include magnifier, narrator and on-screen keyboard which are useful as temporary ways of providing access 
(see, Microsoft). Validation and Repair Tools will help you to check whether your web sites and other web-based content is fully accessible for disabled students. A list of validation tools can be found on the TechDis web site [19]. Multimedia content should be captioned so that it is accessible for deaf students

In the case of provisioning electronic information service for visually impaired persons. personal computers, software and devices such as "Braille touch technology", "sound technology", "Window-Eyes" have been developed; The Vocal-Eyes product which provided speech output from a DOS-based screen [20], and "sight technology" are good examples [11].

\subsection{For Communication and Interaction}

Augmentative /Alternative Communication (AAC) includes the use of eye pointing, gesture, signing, symbols, word boards, or a speech output device. Everyone uses AAC techniques from time to time, but some people depend on them all the time [22].

Computer based AAC systems can be essential aids for SEN in contexts of communication and interaction [23]. Such a system exists to assist people to overcome communication-difficulties. The systems can contain stored communication material for people with impaired communication to retrieve and use during interaction. For example, a person who cannot speak functionally may still have some limited use of speech that allows him or her to communicate simple messages to familiar partners. This individual's speech may then be augmented by the use of gestures, a non electronic communication board, or an electronic voice-output device. The context and content of the message to be communicated would then determine which component(s) the individual used to communicate.

However, researchers also found that, as the size of the stored information corpus in the AAC system increases, the task of searching and retrieving items from that corpus will become more demanding, which will make it less easy for someone to successfully access and use such material, and thus make it more dependent on the individual's needs and skills. Thus, the full potentials of appropriate AAC systems that provide an individual with a multimodal means of communication are yet to be realised. How other children's attitudes towards a peer who used AAC were influenced by type of AAC system/device accessed by the child for communication is also an emerging and important subject to investigate.

Personal support technologies, such as personal digital assistants (PDAs), may have the ability to aid learners with cognitive disabilities [24]. Researchers show that parents or caregivers can pre-program a PDA or desktop software with educational, vocational, or daily living tasks to prompt individuals with cognitive disabilities to perform defined vocational and independent living tasks. Reportedly, specialized PDA software is currently available for enabling individuals with developmental and other cognitive disabilities to manage personal schedules during their work tasks. PDAs may also interface with wireless communication protocols to track and monitor an individual's daily activities, and provide prompts to the individual as needed to complete educational or work tasks [25]. 


\subsection{For Learning}

The meta-synthesis enables us to specifically focus on using ICT in the learning processes.

First, ICT may assist learners with disabilities with empowerment to access to their environment and opportunities for personal development which are otherwise denied them. ICT can be a vital tool in supporting advocacy and self-advocacy for people with learning disabilities and can be the means of bringing marginalized people back into their communities.

Second, assistive technologies (see section 4.1) may include specialized training services, voice interfaces, picture-based email programs, and adapted Web browsers. Wearable intelligent devices may also assist learners. For example, a wearable data glove has been developed that translates Sign Language and transmits this information wirelessly to an electronic display.

Third, personal support technologies (see section 4.2) may benefit individuals in the classroom to remain on task, remind them of pending assignments, and provide access to information on the computer or the Internet. The effectiveness of computer-based learning techniques for students with cognitive disabilities has been documented [26].

\section{Conclusion}

Education should be able to reach the special educational needs of all learners. There is great ICT potential that can be explored to facilitate this challenging task. However, little has been done to develop a methodology bridging ICT and special educational needs. The lack of such a methodology can be one of the main factors that limit the current ICT in developing a systematic construction of a toolkit for a specific population. Many useful enquiries about special educational needs have been conducted by individual use-cases and specific experiments in practical educational settings. Whilst known technical tools such as word-processor, screen-reading, graphics, databases, the internet, and problem-solving software packages in a special need oriented "curriculum" still provide useful snapshots of ICT applications from particular points in time, these pictures now require updating. To do this, the meta-synthesis methodology is a powerful analytical tool for bridging the divide. In particular, the methodology enables ICT developers to think of a disability in the more functional contexts, not merely in a form of abstraction, i.e. the contexts of assistive, sensory, communicative and interactive functions in learning.

\section{References}

1. Lacey, P. and Ouvry, C. (ed.): People with Profound and Multiple Learning Disabilities a collaborative approach to meeting complex needs. London: Fulton (2006)

2. Closs, A.:Education of Children with Medical Conditions. London: David Fulton (2000)

3. Ainscow, M.: Towards inclusive schooling. British Journal of Special Education (1997) 24 (1) 3-6

4. Davis, P., Florian, L.: Teaching Strategies and Approaches for Pupils with Special Educational Needs: A Scoping Study. DfES Research Report No.516 (2004) 
5. Kauffman, J., Hallahan, D.: Special Education: What Its and Why We Need It. MA: Pearson Allyn and Bacon (2006)

6. Becta: http://www.becta.org.uk/

7. Phelan, A., Haughey, E. (ed.): Information \& Advice - Special Educational Needs and Information and Communication Technology. National Centre for Technology in Education, Dublin City University, Ireland (2000)

8. Woodward, J., Gallagher, D., Rieth, H.: The Instructional Effectiveness of Technology for Students with Disabilities. In: Woodward, J. Cuban, L. (ed.): Technology, Curriculum and Professional Development: Adapting Schools to Meet the Needs of Students with Disabilities. Thousand Oaks, CA: Corwin Press (2001)

9. Williams P, Jamali H.R., Nicholas, D.: Using ICT with People with Special Education Needs: What the Literature Tells Us. In: Aslib Proceedings (2006) 58(4) 330-345

10. Mooij, T., Smeets, E.: Modelling and Supporting ICT Implementation in Secondary Schools. Computers \& Education (2001) 36(3) 265-281

11. Jones, A., Tedd, L.: Provision of Electronic Information Services for the Visually Impaired: An Overview with Case Studies from Three Institutions within the University of Wales. Journal of Librarianship and Information Science, (2003) 35 (2) 105-113

12. Gu, J-F., Tan, X-J.: A Test on Meta-synthesis System Approach to Forecasting the GDP Growth Rate in China. In: Proceedings of the 47th Annual Meeting of the International Society for the Systems Sciences, at Hersonissos, Crete (2003) 6-11 July

13. Tang, X-J., Liu, Y.: Computerized Support for Qualitative Meta-synthesis as Perspective Development for Complex Problem Solving. In: International Conference on Creativity and Innovation in Decision Making and Decision Support (CIDMDS 2006) LSE London (2006)

14. Thomas, T.: Renaissance eLearning. Pfeiffer Wiley (2006)

15. Audit Commission (2002) www.audit-commission.gov.uk

16. Dee, L., Byers, R., Hayhoe, H., Maudslay, L.: Enhancing Quality of Life - Facilitating Transitions for People with Profound and Complex Learning Difficulties: a Literature Review. London: Skill/Cambridge: University of Cambridge (2002)

17. Florian, L., Hegarty, J. (ed.): ICT and Special Educational Needs - a tool for inclusion. Open University Press (2004)

18. DfES Special Educational Needs Code of Practice. London: DfES (2001)

19. TechDis (2006) http://www.niad.sussex.ac.uk/subcategory_descriptions.cfm

20. Bowman, V.: Reading between the Lines: an Evaluation of Window-Eyes Screen Reader as a Reference Tool for Teaching and Learning. Library Hi Tech. (2002) 20 (2)162-168

21. Wahl, L.: Assistive technology enhances learning for all (2006) http://www.edutopia.org/php/article.php?id=Art_1045

22. International Society for Augmentative \& Alternative Communication (2006), http://www.isaac-online.org/en/home.shtml

23. Beck, A. R., Parette, P., Baley, R. L.: Multimedia Effectiveness in An AAC Pre-service Setting. Journal of Special Education Technology. (2006) 20(4) 39-49

24. Bergman, M. M.: The Benefits of a Cognitive Orthotic in Brain Injury Rehabilitation. Journal of Head Trauma Rehabilitation. (2002) 17(5) 431-445

25. McDonough, B.: Wearable tech helps disabled students. NewsFactor Network (2002) http://sci.newsfactor.com/perl/story/17419.html

26. Blischak, D. M., Schlosser, R. W. (2003) Use of Technology to Support Independent Spelling by Students with Autism. Topics in Language Disorders. (2003) 23(4) 293-304 